\title{
Bioanalysis
}

\section{The breadth of biomarkers and their assays: part 2}

\author{
"...it is biomarkers that are opening up new vistas and \\ forcing the expansion of existing technology solutions \\ and the exploration of alternatives."
}

First draft submitted: 21 October 2016; Accepted for publication: 25 October 2016; Published online: 18 November 2016

Once again, we are excited to serve as Guest Editors for this second issue of the Special Focus on Bioanalysis of Biomarkers, in Bioanalysis. In this edition, we are pleased to introduce new content that lends additional insights into the central themes explored in the first issue, where we highlighted the range of challenges encountered in the measurement of biomarkers and the breadth of technological solutions being applied to meet those challenges. In the following pages, we present a variety of content that includes commentary, research and review articles that expand upon some common challenges, leverage experience with tried and true technologies, and introduce us to new methods, new technologies and an array of different analytes, which together speak to the complexity of biomarker bioanalysis.

Two topics at the center of many biomarker discussions are accuracy and parallelism [1-4]. It is now widely acknowledged that demonstration of true accuracy is a rarity in the biomarker space. In a Commentary provided here, Rand Jenkins discusses some of the complexities associated with accuracy, the realities of relative accuracy, and provides perspective on the commonalities in this regard between biomarkers and therapeutics with endogenous counterparts [5]. On the parallelism front, Lindsay King's Commentary summarizes and contextualizes key concepts and focuses on the application of parallelism to demonstrate immunological similarity between the calibrator and the endogenous analyte and how this differs from assessment of matrix interference [6]. Amaravadi also provides an editorial article on the general perspective on biomarker measurements, the associated challenges and where we are heading [7].

The use of biomarkers as surrogates for drug-drug interaction studies is an area of growth as pharmaceutical companies search for ways to identify potential side effect risks to clinical study subjects and accelerate the development process. Two papers are presented in this special issue using the familiar UHPLC-MS/MS platform. Using a surrogate matrix, Yuan et al. provide a case example where $7 \alpha$-hydroxy-cholesten-3-one (C4) was measured as a biomarker by UHPLCMS/MS to assess CYP7A1 enzyme activity as it relates to changes in bile acid synthesis [8]. Similarly, Zheng et al. developed a UHPLC-MS/MS assay to quantify the total unconjugated forms of urinary dehydroepiandrosterone (DHEA), 7 $\beta$-hydroxy-DHEA, cortisone and $6 \beta$-hydroxycortisone as potential biomarkers of CYP3A inhibition and induction [9].

The increasing need for improved analysis of protein biomarkers, in particular the quantification of cytokines in biological fluids, has prompted the development of more efficient and more sensitive ligand-binding assay platforms [10]. In this issue, Gupta and colleagues describe the sensitivity, accuracy, precision and other analytical characteristics of multiplexed cytokine assays in plasma using the Simple Plex ${ }^{\mathrm{TM}}$ Ella microfluidic platform, ProteinSimple, CA, USA. Their demonstration of analytical performance, and comparison with traditional ELISA, pave the way for assay implementation in
Mark E Arnold ${ }^{1}$, Hendrik Neubert ${ }^{2}$ \& Lauren $\mathrm{F}$ Stevenson ${ }^{*}, 3$

${ }^{1}$ Covance Laboratories, Inc., 115 Silvia Street Ewing, NJ 08628, USA ${ }^{2}$ Biomedicine Design, Worldwide Research \& Development, Pfizer, Inc., Andover, MA 01810, USA ${ }^{3}$ Global Biomarker Discovery \& Development, Biogen, 225 Binney Street, Cambridge, MA 02142, USA *Author for correspondence: lauren.stevenson@biogen.com 
exploratory clinical biomarker studies, such as those that investigate pharmacodynamic changes resulting from immune checkpoint inhibitor treatment [11].

While extensive activity is seen in the pages of Bioanalysis and other journals on protein and small-molecule biomarker analytes, other chemotypes are being explored as part of mechanistic studies. da Costa et al. provide a review of the potential use of such an alternative analyte, miRNAs, in a clinical setting as circulating biomarkers in Parkinson's disease [12]. miRNAs have the potential for being an early diagnostic of the disease which is normally diagnosed through later stage neuromotor symptoms. In addition to discussing the various technologies employed, the authors note the regulatory authorities' interest and consortia focused on validating the relevance of specific miRNAs as diagnostic biomarkers.

Finally, the measurement of cellular biomarkers by flow cytometry for characterization of the disease state or evaluation of response to treatment is an area of increasing emphasis in drug development. To this end, Sun and colleagues describe a case study where a quantitative flow cytometry assay was developed for the detection of intracellular expression of $\mathrm{Ki} 67$, a cell proliferation marker, in cellular subsets of human blood [13]. Assay validation demonstrates robustness of the method to monitor Ki67 expression in clinical samples from immunotherapy studies.

\section{References}

1 Arnold ME, Booth B, King L, Ray C. Workshop report: crystal city VI-bioanalytical method validation for biomarkers. AAPS J. 11 doi:10.1208/s12248-016-9946-6 (2016) (Epub ahead of print).

2 Ray CA. Biomarker accuracy: exploring the truth. Bioanalysis 6(3), 269-271 (2014).

3 Stevenson LF, Purushothama S. Parallelism: considerations for the development, validation and implementation of PK and biomarker ligand-binding assays. Bioanalysis 6(2), 185-198 (2014).

4 Lee JW, Devanarayan V, Barrett YC et al. Fit-for-purpose method development and validation for successful biomarker measurement. Pharm. Res. 23(2), 312-328 (2006).

5 Jenkins R. Accuracy: a potential quandary in regulated bioanalysis of 'endogenous' analytes. Bioanalysis 8(23), 2393-2397 (2016).

$6 \quad$ King LE. Parallelism experiments in biomarker ligand binding assays to assess immunological similarity. Bioanalysis 8(23), 2387-2391 (2016).

7 Amaravadi L. Biomarker measurements: how far have we come and where are we heading? Bioanalysis 8(23), 2383-2386 (2016).

8 Yuan L, Luo Y, Kandoussi H, Ji QC. A simple, fast, sensitive and robust LC-MS/MS bioanalytical assay for evaluating 7 $\alpha$-hydroxy-4-cholesten-3-one biomarker in a clinical program. Bioanalysis 8(23), 2445-2455 (2016).
The issue also includes the parts two and three of the White Papers to be published following the Workshop on Recent Issues in Bioanalysis annual meeting held in April this year, which focus on biomarker assay validation $[14,15]$.

While the variety of new chemo- and proteo-type drug constructs continue to provide challenges to the bioanalytical scientist, it is biomarkers that are opening up new vistas and forcing the expansion of existing technology solutions and the exploration of alternatives. As part of the scientific community working in this space, the Guest Editors and hopefully the readers, have found the content of both Special Focus Issues on biomarkers inspiring and eliciting a further commitment to being active participants in the development of new therapies for patients with serious diseases.

\section{Financial \& competing interests disclosure}

The authors have no relevant affiliations or financial involvement with any organization or entity with a financial interest in or financial conflict with the subject matter or materials discussed in the manuscript. This includes employment, consultancies, honoraria, stock ownership or options, expert testimony, grants or patents received or pending, or royalties.

No writing assistance was utilized in the production of this manuscript.

9 Zheng N, Christopher LJ, Ma X et al. UHPLC-MS/ MS Bioanalysis of Urinary DHEA, cortisone and their hydroxylated metabolites as potential biomarkers for CYP3A-mediated drug-drug interactions. Bioanalysis 8(23), 2429-2443 (2016).

10 Fischer SK, Joyce A, Spengler M et al. Emerging technologies to increase ligand binding assay sensitivity. AAPS J. 17(1), 93-101 (2015).

11 Gupta V, Davancaze T, Good J et al. Bioanalytical qualification of clinical biomarker assays in plasma using a novel multi-analyte simple Plex ${ }^{\mathrm{TM}}$ platform. Bioanalysis 8(23), 2415-2428 (2016).

12 da Costa AN. Recent developments in circulating biomarkers in Parkinson's disease: the potential use of miRNAs in a clinical setting. Bioanalysis 8(23), 2497-2518 (2016).

13 Sun Y, Yang K, Bridal T, Ehrhardt AG. Robust Ki67 detection in human blood by flow cytometry for clinical studies. Bioanalysis 8(23), 2399-2413 (2016).

14 Song A, Lee A, Garofolo F et al. 2016 White paper on recent issues in Bioanalysis: focus on biomarker assay validation (BAV) (part 2 - hybrid LBA/LCMS and Input from regulatory agencies). Bioanalysis 8(23), 2457-2474 (2016).

15 Richards S, Amaravadi L, Pillutla R et al. 2016 White paper on recent issues in Bioanalysis: focus on biomarker assay validation (BAV) (Part 3 - LBA, Biomarkers and immunogenicity). Bioanalysis 8(23), 2475-2496 (2016). 